\title{
Modeling Tidal Current Speed Using a Wakeby Distribution
}

\author{
Mingjun Liu ${ }^{\mathrm{a}}$, Wenyuan $\mathrm{Li}^{\mathrm{a}}$, Roy Billinton ${ }^{\mathrm{b}}$, Caisheng Wang ${ }^{\mathrm{c}}$ and Juan $\mathrm{Yu}^{\mathrm{a}}$
}

a: State Key Laboratory of Power Transmission Equipment \& System Security and New Technology, College of Electrical Engineering, Chongqing University, Chongqing, China

b: University of Saskatchewan, Saskatoon, Canada

c: Wayne State University, Detroit, USA

Email address of Corresponding author: Wenyuan.li@ieee.org

Abstract - Considerable interest in tidal power is being driven by the global push for renewable energy. The first step in modeling tidal energy sources in power systems analysis is to determine an appropriate probability distribution for tidal current. This paper proposes a Wakeby distribution to model the probabilistic characteristics of tidal current speed. The Wakeby distribution and nine other popular distributions are investigated using four years of tidal current speed data at ten different sites. Comparisons are conducted using the Kolmogorov-Smirnov test (K-S test) and the root-mean-square error (RMSE) index for both posteriori and priori tests. The results verified that the Wakeby distribution not only has the best statistical performance but also is the only one of the ten selected distributions that passed the K-S test.

Keywords - Probabilistic model, tidal current speed, tidal power, Wakeby distribution

\section{Introduction}

Climate change has a significant impact on the earth's natural systems and on human being life styles. People in their daily lives have primarily relied for many years on the utilization of fossil fuels in electric power supply. Finding clean and renewable energy is one way to deal with global warming. Considerable interest in ocean energy is being driven by the global push for renewable energy [1-5].

The probabilistic modeling of wind energy has drawn considerable attention in the past decade and extensive research work has been published. To our knowledge, however, relatively limited research effort has been devoted to the probabilistic modeling of tidal current energy and its application in power system analysis. Tidal current data show that 
tidal current speeds vary every minute, every hour and every day due to many reasons including turbulent, surges, wind-driven flows and other random factors. Although many engineering projects are under way, little work has been reported on studies of the statistical characteristics of tidal current. As in wind or solar energy analyses, a probabilistic model of tidal current speed is the basis for probabilistic analysis of a power system with tidal energy sources. Existing references on tidal energy are focused on deterministic analysis techniques [6-8]. These techniques are very useful but a probabilistic model is required to capture the random features of tidal current speed. One tidal current prediction in use is based on a simple least squares method for only a short term (at most monthly) [8]. Such a method can provide a predicted sample of tidal current speed in the form of a single value (such as the mode speed of the tidal current) but cannot estimate the uncertainty associated with a predicted value as it does not provide a complete picture of the tidal current speed random variability and therefore cannot be used for the purpose of long term prediction in system planning studies.

Modeling the randomness of wind speed is an important and well known requirement in wind source related planning, operation and analysis of power systems. Similarly, establishing an appropriate probability distribution model for tidal current speed is the first step in random behavior analysis of tidal power, tidal energy estimation, and tidal power source related system planning and operation studies, such as probabilistic power flow, probabilistic voltage and transient stability studies, and reliability evaluation. Unfortunat

ely, an extensive literature search did not reveal any published references in this regard. Weibull, Rayleigh, Inverse Gaussian, Burr, Lognormal and Beta distributions are the most commonly used distributions in wind speed or solar insolation modeling and relevant power system analyses [9-10]. Among these distributions, the three-parameter Weibull model is the most popular. In hydrology, Generalized Extreme Value, Gama family and Wakeby distributions are routinely used in flood frequency and rainfall analyses [11-12]. A natural question therefore is: can any of these probability distributions be used to model tidal current speed and to characterize tidal power sources in power system analysis? 
The objective of this paper is to establish an appropriate probability distribution for tidal current speed so that the random nature of tidal energy can be modeled in subsequent applications in power system planning and operation containing tidal power sources. The paper investigates the fitness of the distributions noted earlier to tidal current speed at ten different sites in North America with a focus on the Wakeby distribution. One challenge is that, unlike other distributions, a Wakeby distribution does not have an explicit cumulative or density probability distribution function but is expressed by a quantile function with five parameters. The most popular maximum likelihood estimation method suitable for parameter estimation of other distributions does not work for the Wakeby distribution. The L-moment estimation method is proposed in this paper to tackle this difficulty. Three different statistical test techniques are presented and both posteriori and priori test methods are used to select the most appropriate probability distribution. The results indicate that the Wakeby distribution is the best distribution for tidal current speed. It successfully passed the Kolmogorov-Smirnov (K-S) test for the tidal current speed data at all ten different sites, whereas other distributions failed in the tests. Compared to other distributions, the Wakeby distribution also reached the minimum root-mean-square error (RMSE) in both posterior and priori tests, which means that there is the smallest gap between the actual tidal current speed data and the Wakeby distribution not only for past behavior but also for predictive purposes.

This paper is arranged as follows. A Wakeby distribution and its parameter estimation method for tidal current are given in Section 2. Other distributions used for comparison purposes are summarized in Section 3. In Section 4, three statistical test methods for selecting the best distribution for tidal current speed are presented. Statistical test results and analyses using the K-S test and both posteriori and priori tests are shown in Section 5 to demonstrate the effectiveness of the Wakeby distribution in modeling tidal current speed, followed by the conclusions in Section 6.

\section{Wakeby distribution and its parameter estimation}

The Wakeby distribution (WAK) was defined by Thomas and first introduced by Houghton [13] in hydrology. Following Houghton, other researchers employed the 
Wakeby distribution for flood frequency and rainfall analyses [14-17], especially for modeling extreme events. Mathematically, the Wakeby distribution has the following properties:

1) The Wakeby distribution with five parameters can mimic a skewed distribution form, which is generally true for tidal current speed because of the turbulent and surge components;

2) It attains a wider range of distributional shapes than commonly used distributions and thus is extremely useful for analyzing the data of tide current speed that has distinct distribution forms at different geographical locations;

3) It can model large outliers thanks to its heavy upper tail, which is the case for tidal current speed data;

4) It has a lower bound and is appropriate for a dataset of tidal current speed that has a minimum value.

\subsection{Definition}

The Wakeby distribution is considerably different from other distributions as it does not have an explicit cumulative or density probability distribution function. The five-parameter Wakeby distribution is defined by the following quantile function [18]:

$$
x(F)=\xi+\frac{\alpha}{\beta}\left[1-(1-F)^{\beta}\right]-\frac{\gamma}{\sigma}\left[1-(1-F)^{-\sigma}\right]
$$

where $F \equiv F(x)=P(X \leq x)$ is the cumulative probability function (CPF) without an analytical expression; $x(F)$ is a quantile value corresponding to a given $F$ value; $\xi$ is the location parameter; $\alpha$ and $\beta$ are the scale parameters; $\gamma$ and are the shape parameters.

The range of $x$ is given by:

$$
\left\{\begin{array}{l}
\xi \leq x \leq \infty \quad \text { if } \sigma \geq 0 \text { and } \gamma>0 \\
\xi \leq x \leq \xi+\frac{\alpha}{\beta}-\frac{\gamma}{\sigma} \quad \text { if } \sigma<0 \text { or } \gamma=0
\end{array}\right.
$$

Taking derivatives with regard to $x$ at both sides of (1) yields: 


$$
1=\left[\alpha(1-F)^{\beta-1}+\gamma(1-F)^{-\sigma-1}\right] \frac{d F}{d x}
$$

Since $d F / d x=f(x)$, the probability density function (PDF) of the Wakeby distribution can be expressed by:

$$
f(x)=\left[\alpha(1-F)^{\beta-1}+\gamma(1-F)^{-\sigma-1}\right]^{-1}
$$

where $F$ is the cumulative probability function as shown in $(1) ; f(x)$ is the probability density function. Although there is no analytical expression for $F$, it can be estimated using the sample data of tidal current speed and in turn $f(x)$ can be calculated. This equation is used in Sections 4 and 5 to calculate the RMSE index.

\subsection{Parameter estimation}

As mentioned earlier, the Wakeby distribution has no analytical cumulative or density distribution function. Therefore, the maximum likelihood estimation method, which is appropriate for other distributions, cannot be applied to estimate the parameters of a Wakeby distribution. An L-moment estimation (LME) method [19] is used to estimate the five parameters of the Wakeby distribution. The basic idea is to estimate the parameters by calculating the first five linear moments from sample data and let each of them be equal to the corresponding moment expression that can be derived from the definition of L-moment. The L-moment is defined by [19]:

$$
\lambda_{r}=\int_{0}^{1} x(F) L p_{r-1}(F) d F
$$

where

$$
\operatorname{Lp}_{r}(F)=\sum_{k=0}^{r} l p_{r, k} F^{k}
$$

and

$$
l p_{r, k}=(-1)^{k}\left(\begin{array}{l}
r \\
k
\end{array}\right)\left(\begin{array}{c}
r+k \\
k
\end{array}\right)
$$

where $\operatorname{Lp}_{r}(F)$ is the $r$ th shifted Legendre polynomial.

Substituting (1) into (5), the first five L-moments of the Wakeby distribution can be 
derived as follows:

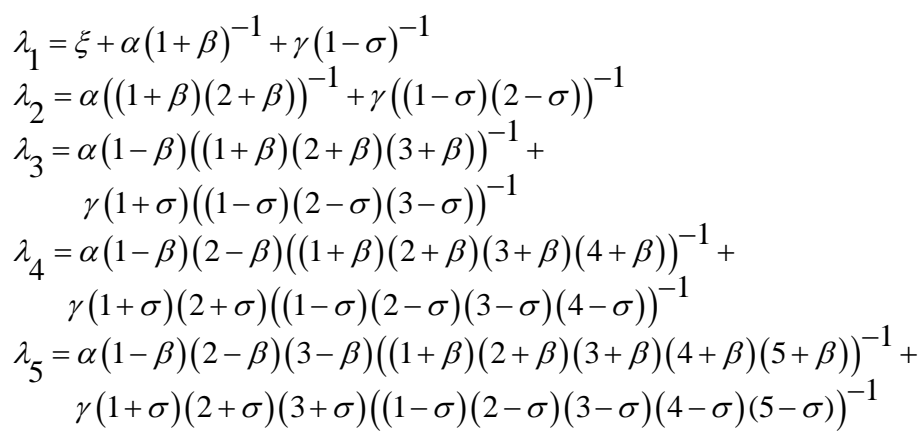

On the other hand, sample-based L-moments can be calculated from historical records of tidal current speed data. For a given sample size $n$, let $x_{1}, x_{2} \cdots x_{n}$ be a set of samples of tidal current speeds with the increasing order $x_{1} \leq x_{2} \leq \cdots \leq x_{n}$. The $r$ th sample-based L-moment can be calculated by:

$$
l_{r}=\left(\begin{array}{c}
n \\
r
\end{array}\right)^{r} \sum_{1 \leq i_{1}<i_{2}<\cdots<i_{r} \leq n} \sum_{k=0}^{-1} \sum_{k=1}^{r-1}(-1)^{k}\left(\begin{array}{c}
r-1 \\
k
\end{array}\right) x_{i_{r-k: n}}, r=1,2, \cdots n
$$

The first five sample-based L-moments can be calculated from (9) as follows:

$$
\begin{aligned}
& l_{1}=n^{-1} \sum_{i} x_{i} a \\
& l_{2}=\frac{1}{2}\left(\begin{array}{l}
n \\
2
\end{array}\right)^{-1} \sum_{i>j}^{\sum \sum}\left(x_{i: n}-x_{j: n}\right) \\
& l_{3}=\frac{1}{3}\left(\begin{array}{l}
n \\
3
\end{array}\right)^{-1} \sum_{i>j>k}^{\sum \sum \sum}\left(x_{i: n}-2 x_{j: n}+x_{k: n}\right) \\
& l_{4}=\frac{1}{4}\left(\begin{array}{l}
n \\
4
\end{array}\right)^{-1} \sum_{i>j>k>l}^{\sum \sum \sum}\left(x_{i: n}-3 x_{j: n}+3 x_{k: n}-x_{j: n}\right) \\
& l_{5}=\frac{1}{5}\left(\begin{array}{l}
n \\
5
\end{array}\right)^{-1} \quad \sum_{i>j>k>\sum \sum}\left(x_{i: n}-4 x_{j: n}+6 x_{k: n}-4 x_{j: n}+x_{m: n}\right)
\end{aligned}
$$

Once $l_{r}(r=1, \ldots 5)$ are obtained from tidal current speed data, use $l_{r}=\lambda_{r}(r=1, \ldots 5)$ in (8) to build the five simultaneous equations. The Newton-Raphson method can be used to solve this set of equations to obtain the five parameters of the Wakeby distribution: $\xi$, $\alpha, \beta, \gamma$ and $\sigma$. 


\section{Other distributions in the comparisons}

In order to select a suitable distribution for tidal current speed, other possible distributions should be considered and compared with the Wakeby distribution. The parametric distributions used in modeling wind speed or solar insolation include the three-parameter Weibull distribution (Wei-3), Rayleigh distribution (Ray), three-parameter Inverse Gaussian distribution (IG-3), Burr distribution, Lognormal distribution $(\log N)$ and Beta distribution. These distributions manifest relatively good fitness to wind speed or solar insolation data depending on the wind speed or solar insolation characteristics at different sites $[9,10,20]$. Among these distributions, the three-parameter Weibull distribution is the most popular one used in modeling wind speed. A kernel density estimate method, as a nonparametric model, has a better overall performance and adaption for various datasets in estimating long term wind speed probability distributions [20]. Considering the similarity in randomness between wind speed/solar insolation and tidal current speed, it is logical to also consider these distributions for comparison purposes. In addition, the Generalized Extreme Value (GEV) and three-parameter Generalized Gama distributions (GG-3) are both frequently used in hydrological studies and therefore they are also considered in the comparison analyses.

The probability density functions (PDF) of each parametric distribution employed in this paper are given in Table 1. Using the analytical expressions, the parameters of the distributions can be easily estimated using the maximum likelihood estimation (MLE) method. The details of the MLE technique can be found in a relevant statistics literature or book [21]. The Kernel Smooth Density (KSD) estimate is a non-parametric estimation method and no parameter estimation is required. The best bandwidth, however, needs to be determined [20]. The KSD estimate listed in Table 1 is a Gaussian kernel smooth function.

Table 1 is here

\section{Statistical test methods}

The following three test methods are used in Section 5 as the selection criteria in order 
to better understand the performance of each distribution and to select the best distribution for tidal current speed from the Wakeby distribution discussed in Section 2 and the other nine distributions given in Table 1.

\subsection{Kolmogorov-Smirnov test}

The Kolmogorov-Smirnov test (K-S test) is a method used to judge if a sample comes from a population with a hypothesized continuous distribution. The K-S test measures the distance between the empirical cumulative distribution function (ECDF) of samples and the cumulative distribution function of a hypothesized theoretical distribution [9]. For an ordered tidal current speed sample sequence $x_{1}, x_{2} \cdots x_{n}$ (from the smallest to the largest) following some distribution with a cumulative distribution function $F(x)$, the ECDF is defined by

$$
F_{n}(x)=\frac{1}{n} \sum_{i=1}^{n} I\left(x_{i}\right) ; I\left(x_{i}\right)= \begin{cases}1 & x_{i} \leq x \\ 0 & x_{i}>x\end{cases}
$$

The K-S statistic $\left(D_{n}\right)$ for a given $F(x)$ is calculated by

$$
D_{n}=\sup _{x}\left|F_{n}(x)-F(x)\right|
$$

A tested distribution is rejected if the test statistic $\left(D_{n}\right)$ is greater than the critical value at a given significance level, which is often selected to be 0.05 . Otherwise, if $D_{n}$ is smaller than the critical value, the tested distribution is accepted. The K-S test shows how well a tested distribution fits the observed dataset as an entire shape. This method is used to test the goodness-of-fit of the ten distributions for tidal current speeds at ten different sites located in North America in Section 5.2.

\subsection{Root mean square error}

The K-S test focuses on the shape of a whole distribution. It does not provide information on the match degree on a point-to-point basis. The root mean square error (RMSE) is defined as the mean error between the histogram and theoretical probability density distribution of tidal current speed data. The root mean square error is calculated by: 
$R M S E=\left[\frac{1}{I_{N}} \sum_{i=1}^{I_{N}}\left(\hat{f}\left(x_{i}\right)-f\left(x_{i}\right)\right)^{2}\right]^{\frac{1}{2}}$

where $\hat{f}\left(x_{i}\right)$ is the probability in the ith interval of the theoretical density distribution, $f\left(x_{i}\right)$ is the probability in the $i$ th interval of a histogram of tidal current speed data, and $I_{N}$ is the number of intervals.

Obviously, a smaller value of RMSE represents a better fit of a tested distribution to the actual tidal current speeds. This index is used for both posteriori and priori tests [22] in Sections 5.3 and 5.4. A posteriori test in the paper refers to a case in which a few years of historical tidal current speed data are used to estimate the parameters of a theoretical probability distribution and the RMSE index is calculated using the same historical data and the theoretical distribution. A priori test in the paper refers to a case in which only parts of the historical tidal current speed data are used to estimate the parameters of a theoretical probability distribution and the RMSE index is calculated using the remaining data records, which can be viewed as hypothetic future samples, and a set of tidal current speed data randomly sampled from the estimated theoretical distribution. The results of posteriori comparisons are given in Section 5.3 and those of priori comparisons in Section 5.4.

\subsection{Sampling theoretical distribution}

A set of tide current speeds needs to be randomly sampled from a theoretical distribution in order to conduct a priori test. The inverse transform method (ITM) is used to generate samples of tidal current speed following a theoretical distribution in this paper. The ITM is based on the proposition that if a random variate $R_{v}$ follows a uniform distribution in the interval between $[0,1]$, the random variate $X=F^{-1}\left(R_{v}\right)$ has a continuous cumulative probability distribution function $F(x)[10]$.

The first step to generate a random variate sequence using the inverse transform method is to generate a uniformly distribute random number sequence $R_{v}$ in $[0,1]$. The random variate can then be calculated by $X=F^{-1}\left(R_{v}\right)$. 
It can be seen from (1) that the five-parameter Wakeby distribution is given in the form of $X=F^{-1}\left(R_{v}\right)$, that is:

$$
X=\xi+\frac{\alpha}{\beta}\left[1-\left(1-R_{v}\right)^{\beta}\right]-\frac{\gamma}{\sigma}\left[1-\left(1-R_{v}\right)^{-\sigma}\right]
$$

It is therefore relatively straightforward to use the ITM to create samples of tidal current speed following the Wakeby distribution. It can be seen from the results in Section 5.3 that the generalized extreme value (GEV) distribution and three-parameter Weibull (Wei-3) distribution provide root-mean-square error indices that are secondary to those provided by the Wakeby distribution, but are better than those provided by any other parametric distribution in the posteriori test. A further priori comparison is conducted among the Wakeby, generalized extreme value and three-parameter Weibull distributions in Section 5.4. In order to do this, it is necessary to generate the random samples of tidal current speed following the generalized extreme value and three-parameter Weibull distributions.

The cumulative probability distribution function of the generalized extreme value distribution is:

$$
F(x)=\exp \left(-(1+k z)^{-1 / k}\right), \quad z \equiv \frac{x-\mu}{\sigma}
$$

where $k, \sigma$ and $\mu$ are the shape, scale and location parameters, respectively. Letting a uniform distribution random number $R_{v}$ equal the generalized extreme value cumulative probability distribution function yields:

$$
R_{v}=F(x)=\exp \left(-(1+k z)^{-1 / k}\right)
$$

Equivalently,

$$
X=\mu+\frac{-\sigma+\sigma\left(-\ln R_{v}\right)^{-k}}{k}
$$

where $X$ is a sample of tidal current speed following the generalized extreme value distribution. Similarly, letting a uniform distribution random number $R_{v}$ equal the three-parameter Weibull cumulative probability distribution function gives: 


$$
R_{v}=F(x)=1-\exp \left(-\left(\frac{x-\gamma}{\beta}\right)^{\alpha}\right)
$$

where $\alpha, \beta$, and $\gamma$ are the shape, scale and location parameters, respectively. Using the inverse transform method yields:

$$
X=\gamma+\beta\left[-\ln \left(1-R_{v}\right)\right]^{1 / \alpha}
$$

Since $\left(\begin{array}{ll}1 & R_{v}\end{array}\right)$ distributes uniformly in the same way as $R_{v}$ in the interval[0,1], (19) can be rewritten as:

$$
X=\gamma+\beta\left[-\ln \left(R_{v}\right)\right]^{1 / \alpha}
$$

The algorithm for generating the Wakeby, generalized extreme value, or three-parameter Weibull distribution random variate is as follows:

Step 1: Generate a uniform distribution random number sequence $R_{v}$ in $[0,1]$.

Step 2: Calculate a random variate sequence $X$ of tidal current speed using (14), (17), or (20).

The method presented here is used in Section 5.4 to generate a random variate sequence following each of the three distributions noted above.

\section{Result and analysis}

\subsection{Data sources}

Tidal current speed datasets at ten different sites located in North America were studied in this paper. The tidal current speed data were obtained from the National Ocean Service (NOS) of the National Oceanic and Atmospheric Administration (NOAA), which is a scientific agency under the United States Department of Commerce. The NOAA's Center for Operational Oceanographic Products and Services (CO-COPS) and its predecessors have gathered oceanographic data along American coasts for over 200 years. Serving both public and other government agencies, CO-OPS is an authoritative source for accurate, reliable, and timely tidal current measurements.

Starting on January $1^{\text {st }}, 2010$ and ending on December $31^{\text {st }}, 2013$, the four years of tidal current speed data with an interval of one hour (i.e. 24 points each day) were used to study the statistical characteristics of tidal current speeds at the ten sites. Both flood and 
ebb data are taken into consideration. Since tidal current has two stages of flood and ebb, the data were divided into two sets: flood current speeds and ebb current speeds.

Each site's code, name, geographical information, and sample statistics for the maximum speed and mode speed (the value most frequently occurring in a flood or ebb current speed set) are summarized in Table 2.

Table 2 is here.

\section{$5.2 \mathrm{~K}-\mathrm{S}$ test results}

The comparisons in goodness-of-fit between the nine distribution models and the Wakeby distribution for the tidal current speeds at the ten sites were performed using the Kolmogorov-Smirnov test given in Section 4.1. The critical values (indicated by C-Value) and statistic values of each distribution model for the ten sites are summarized

in Table 3. Table 3 also provides the rank order of each distribution in terms of goodness-of-fit in the K-S test. The rank order is shown by the numbers in the brackets with the number (1) indicating the best fitting distribution and the number (10) indicating the worst one. In other words, a smaller statistic value represents a better goodness-of-fit. The F and $\mathrm{E}$ in the second column indicate flood and ebb current speeds, respectively.

\section{Table 3 is here.}

It can be seen that the Wakeby distribution is not only the best distribution in the comparison but also the only distribution that passed the test since the statistic value of only the Wakeby distribution is smaller than the critical value. Although the Kernel Smooth Density and Generalized Extreme Value distributions are ranked in the second and third places, both failed to pass the K-S test. The Lognormal distribution is the worst distribution, whereas the rank orders of other distributions vary from one site to another.

\subsection{Posteriori comparisons between histograms and theoretical probability distributions}

The K-S statistical test provides information on goodness-of-fit for each distribution model of tidal current speed from the viewpoint of whole distribution shape. It cannot, 
however, provide a comparison on a point-to-point basis between the observed data and a probability distribution. A histogram can be calculated from the observed tidal current speed data, whereas the probability density distribution of tidal current speed can be obtained from the assumed theoretical distribution. As a posteriori test, a comparison between the histogram and theoretical probability distributions can be performed using both quantified RMSE indices and visualized distribution curves.

The RMSE values of all the ten distributions (including the Wakeby distribution) at the ten different sites are summarized in Table 4 . The number in the brackets indicates the rank number of the RMSE in an increasing order, and F and E represent the flood and ebb current speeds, respectively. It can be observed from Table 4 that the Wakeby distribution always performs the best for the tidal current speeds at the ten sites since it has the smallest RMSE value, whereas the Beta distribution is the poorest one for all the ten sites. It is interesting to note from Table 3 and Table 4 that the rank order in terms of the K-S test and the RMSE index are not the same. This is understandable because the K-S test focuses on the overall shape of a distribution while the RMSE emphasizes the average error due to differences at all points between the observed tidal current speeds and theoretical distribution. This indicates that a single test method is generally not sufficient. It should be noted that the Wakeby distribution is ranked as the top one in all the cases (at all the sites and for both flood and ebb current speeds) in terms of both the K-S test and RMSE indices.

Table 4 is here.

Because of limitations of length, only four selected figures are shown in Figures 1-4, which present the histograms from the actual tidal current speed data and probability density function (PDF) curves at sites 4, 6, 8 and 9. In these figures, the selected PDFs are those of the Wakeby distribution (the best performance distribution in both the K-S test and RMSE indices), generalized extreme value distribution (the second best distribution in the RMSE index), kernel smooth density estimate (the second best distribution in the K-S test), Lognormal distribution (the poorest performance distribution in the K-S test) and Beta distribution (the poorest performance distribution 
in the RMSE index).

Figure 1, Figure 2, Figure 3 and Figure 4 are here.

It can be seen from the figures that the Wakeby distribution density function is the closest to the histogram of the actual tidal current speed data. The kernel smooth density (KSD) estimate looks to be a possible match for the histogram. However, this is not really true as the KSD has some negative values at the left tail, which is unrealistic because tidal current speed is always greater than or at least equal to zero in real life. This misrepresentation may result in difficulties or errors when applying the KSD formula to tidal power modeling. It is worth noting again that all the other probability distribution models including the KSD did not pass the K-S test except for the Wakeby distribution. An interesting phenomenon observed from Figures 1-4 is that the shapes of the histograms in different cases (for flood or ebb current speeds or at different sites) are different from each other. As expected, the Wakeby distribution can match all the histograms since it can represent a wide range of skewed shapes of tidal current speed. The figures of the histograms and PDF curves at other sites and for other distributions, which could not be included in the paper due to space limitations, demonstrate similar observations and conclusions.

\subsection{Priori comparisons between histograms and theoretical probability distributions}

The posteriori comparisons in Section 5.3 provide information on the gaps between the histograms and the theoretical probability distributions for the tidal current speeds. One deficiency is that the posteriori test does not provide a strong verification on whether a theoretical probability distribution can be used for long term prediction purposes in power system planning analysis. The priori comparison method described in Section 4.2 serves such a purpose. A priori test was performed using the following procedure: the first two years' tidal current speed data were used to estimate the parameters of a theoretical distribution; then the Monte Carlo simulation method given in Section 4.3 was used to generate a random sample sequence, which can represent a pattern of tidal current speed in the future; the actual tidal current speed data in the remaining two years of record and the sampled sequence from the theoretical distribution was compared 
using the RMSE between them.

In order to better understand the good performance of the Wakeby distribution in the priori test, the three-parameter Weibull and generalized extreme value distributions were also considered since they have better RMSE indices in the posteriori test than the other parametric distributions, except the Wakeby distribution. The RMSE indices of the three distributions in the priori test are summarized in Table 5. The histograms sampled from the Wakeby distribution and actual tidal current speed data at site 10 are shown in Figures 5 and 6 . In each figure, the black histogram is based on the sampled speeds from the Wakeby distribution, and the white histogram is based on the actual current speed data in the remaining two years that are not used in the parameter estimation.

Table 5, Figure 5 and Figure 6 are here.

The following observations can be made: 1) The Wakeby distribution has the smallest RMSE values for all the ten sites compared to the other two distributions in the priori test. 2) The RMSE values of the Wakeby distribution in the flood and ebb stages are basically the same, indicating that the Wakeby distribution can flexibly fit the tidal current speeds regardless of flood or ebb current patterns. 3) The RMSE value of GEV or Wei-3 is about 30-66 times larger than that of WAK, and all the RMSE values of WAK are between 0.0011 and 0.0017 . The tidal current speeds randomly sampled from the Wakeby distribution can perfectly match the actual tidal current speed data in the priori test and thus can be used for long term prediction purposes in power system planning studies in addition to other applications (such as probabilistic power flow and reliability analysis). The histograms of sampled and actual tidal current speeds for flood and ebb stages only at site 10 are provided in Figures 5 and 6 due to space limitations. The histograms for the priori tests of tidal current speed data at other sites are similar. It can be concluded from the results in Sections 5.2, 5.3 and 5.4, which were obtained using the three statistical test methods, that the Wakeby distribution is the best distribution fitting tidal current speeds for both flood and ebb stages at different geographical locations. Although the generalized extreme value distribution and three-parameter generalized Gama distribution are widely used in hydrology for rainfall 
and flood frequency analyses, they are not appropriate models for tidal current speeds. The distributions popularly used for modeling wind speeds in power system analysis, including Weibull, Burr, Rayleigh, Inverse Gaussian and Lognormal distributions, do not perform as well for tidal current speeds as they do for wind speeds. The Beta distribution, which is generally accepted for modeling solar insulations, does not work for tidal current speeds at all. The statistical characteristics of tidal current speed are totally different from those of flood frequency, rainfall, wind speed, or solar insolation, although all of them behave randomly.

\section{Further Notes}

Up to now, the tidal current speed has been modeled only by deterministic methods. With the Wakeby distribution of tidal current speed presented in this paper, many different probabilistic analyses can be performed to model the random behavior of tidal power in system planning and operation studies. A straightforward application is to evaluate the probability distribution of tidal power generation using the Wakeby distribution of tidal current speed and the output characteristic of tidal generator. This work has been well achieved and reported in [23]. The results indicate that a deterministic average tidal current speed model or a mode speed model can lead to significant errors in the calculation of tidal power compared to the probabilistic model of tidal generation. Such a probabilistic model can also provide a full picture of tidal power values at different probability levels. More details can be found in Reference [23].

Another application is the reliability assessment of a tidal generation system or power system with tidal sources considering the Wakeby distribution of tidal current speed. It is found by our further investigations that the failure rates of electronic components of tidal power generation system strongly depend on tidal current speed and its probability distribution. In other words, the probability distribution of tidal current speed has a significant impact on the reliability of a tidal generation system. The new findings will be published in subsequent papers.

The probability distribution of power output of a tidal power generator can be used as 
the input information for other probabilistic analyses of power systems, including probabilistic power flow and probabilistic stability analyses. Therefore, the Wakeby distribution of tidal current speed can find more and wider applications in the future.

\section{Conclusions}

This paper proposes a Wakeby distribution model for tidal current speed probability distribution. Creating such a model is the first step in the random behavior analysis of tidal power, tidal energy estimation, and tide power related system planning and operation studies including probabilistic power flow, probabilistic stability analyses and reliability evaluation. The main advantage of this distribution is that it can mimic a wide range of skewed tidal current speed distributions. It can fit both flood and ebb current speeds at different sites with the smallest RMSE index in both posteriori and priori tests and passes the K-S test. This ability indicates that the probabilistic nature of tidal energy can be modeled using the Wakeby distribution in various applications in system planning and operation of power systems with tidal energy sources. The priori test also indicates that the Wakeby distribution can be used for the purpose of tidal current speed uncertainty prediction in long term planning.

The paper examines the appropriateness of ten different distributions in modeling tidal current speed. Four years of actual tidal current speed data at ten different sites with different tidal current behaviors were used in the investigation. The comparisons were performed using the K-S test for goodness-of-fit and the root-mean-square error indices for both posteriori and priori tests between histograms and theoretical probability density distributions. The statistical test results indicate that the Wakeby distribution is the most suitable distribution for tidal current speed. Other examined distributions, which have been accepted in modeling flood frequency, rainfall, wind speed or solar insolation, do not perform well in modeling tidal current speed. All of them did not pass the K-S test and have much worse RMSE indices than those for the Wakeby distribution. This finding is important and useful when a probabilistic model for tidal power is used in the system analysis of power systems with tidal sources.

\section{Acknowledgement}


The work in the paper is partially supported by the National "111" Project of China (B08036), the National Natural Science Foundation of China (Project No. 51177178) and the National Science Foundation of USA under Grant ECCS-1202133.

\section{References}

[1] J. Huckerby, H. Jeffrey and B. Jay, An international vision for ocean energy, OES-IEA, October, 2011, [Online] Available at: http://www.ocean-energy-systems.org

[2] D. Levitan, First Tidal Power in U.S. Starts Flowing to the Grid, IEEE Spectrum, September, 2012, [Online] Available at:

http://spectrum.ieee.org/energywise/green-tech/geothermal-and-tidal/first-tidal-pow er-starts-flowing-to-the-grid

[3] J. Hsu, Tidal Energy Could Supply Half of Scotland's Power, IEEE Spectrum, January, 2014, [Online] Available at:

http://spectrum.ieee.org/tech-talk/green-tech/geothermal-and-tidal/tidal-energy-coul d-supply-half-of-scotlands-power

[4] D. Levitan, Tidal Power Coming to India, IEEE Spectrum, January, 2011, [Online] Available at:

http://spectrum.ieee.org/energywise/green-tech/geothermal-and-tidal/tidal-power-co ming-to-india

[5] S. Hall, China's New Wager: Pulling Energy From the Ocean, The Wall St. Journal, USA, March, 2014, [Online] Available at: http://online.wsj.com/news/articles/SB100014240527023032878045794469040694 $\underline{62752 ? \bmod =\mathrm{LS} 1}$

[6] S.A. Thorpe, An Introduction to Ocean Turbulence, Cambridge University Press, 2007

[7] A. Khaligh and O. G. Onar. Energy Harvesting: Solar, Wind, and Ocean Energy Conversion Systems, CRC Press, 2010

[8] John Boon, Secrets of the Tide: Tide and Tidal Current Analysis and Applications, Storm Surges and Sea Level Trends, Horwood Publishing Limited, 2004

[9] V. L. Brano, A. Orioli, G. Ciulla and S. Culotta, "Quality of wind speed fitting distributions for the urban area of Palermo, Italy", Renewable Energy, vol. 36, no. 3, 2011, pp: 1026-1039

[10]Wenyuan Li, Risk Assessment of Power Systems: Models, Methods, and Applications, 2nd edition, IEEE Press and John Wiley, 2014

[11]K Hamed, and A. R. Rao, Flood Frequency Analysis, Boca Raton, CRC press, 1999

[12]T. Öztekin. "Estimation of the parameters of Wakeby distribution by a numerical least squares method and applying it to the annual peak flows of Turkish rivers", Water Resources Management, vol. 25, no. 5, 2011, pp: 1299-1313.

[13]J. C. Houghton, "Birth of a parent: The Wakeby distribution for modeling flood flows", Water Resources Research, vol. 14, no. 6, 1978, pp: 1105-1109 
[14]T Öztekin, "Wakeby distribution for representing annual extreme and partial duration rainfall series", Meteorological Applications, vol. 14, no. 4, 2007, pp: 381-387.

[15]B. Su, Z. W. Kundzewicz and T. Jiang, "Simulation of extreme precipitation over the Yangtze River Basin using Wakeby distribution", Theoretical and Applied Climatology, vol. 96, no. 3-4, 2009, pp: 209-219

[16]G. A. Griffiths, "A theoretically based Wakeby distribution for annual flood series", Hydrological Sciences Journal, vol. 34, no. 3, 1989, pp: 231-248

[17]T. Haktanir, "Comparison of various flood frequency distributions using annual flood peaks data of rivers in Anatolia", Journal of Hydrology, vol. 136, no. 1, 1992, pp: $1-31$

[18]J. R. M. Hosking and J. R. Wallis, Regional Frequency Analysis: An Approach Based on L-moments, Cambridge University Press, Cambridge, 2005

[19]J. R. M. Hosking, "L-moments: analysis and estimation of distributions using linear combinations of order statistics", Journal of the Royal Statistical Society Series B (Methodological), vol. 52, no. 1, 1990, pp: 105-124

[20]Z. Qin, W. Li and X. Xiong. "Estimating wind speed probability distribution using kernel density method", Electric Power Systems Research, vol. 81, no. 12, 2011, pp: 2139-2146

[21]D. Kececioglu. Reliability Engineering Handbook, DEStech Publications, Inc, 2002

[22]J. S. Baehr, A Priori and A Posteriori, Loyola Marymount University, USA, October, 2006, [Online] Available at: http://www.iep.utm.edu/apriori/

[23]Mingjun Liu, Wenyuan Li, Roy Billinton, Caisheng Wang and Juan Yu, "Probabilistic modeling of tidal power generation," IEEE PES General Meeting, 2015, Denver, July 26-30, 2015 
Tables

Table 1 Probability density functions of various distributions

\begin{tabular}{|c|c|}
\hline Distribution & Probability Density Function \\
\hline KSD & The type of kernel smoother to use: $(\sqrt{2 \pi})^{-1} \exp \left(-u^{2} / 2\right), u=\left(x-X_{i}\right) / h$ \\
\hline GEV & $\exp \left(-(1+k z)^{-1 / k}\right)(1+k z)^{-1-1 / k} / \sigma, 1+k z>0, z=(x-\mu) / \sigma$ \\
\hline GG-2 & $\left(k x^{k \alpha-1} /\left(\beta^{k \alpha} \Gamma(\alpha)\right)\right) \exp \left(-(x / \beta)^{k}\right), 0 \leq x<+\infty$ \\
\hline Wei-3 & $(\alpha / \beta) z^{\alpha-1} \exp \left(-z^{\alpha}\right), z \equiv(x-\gamma) / \beta, \gamma \leq x<+\infty$ \\
\hline Ray & $\left(x / \sigma^{2}\right) \exp \left(-0.5(x / \sigma)^{2}\right), 0 \leq x<+\infty$ \\
\hline IG-3 & $\left(\left(\lambda /\left(2 \pi(x-\gamma)^{3}\right)\right)\right)^{0.5} \exp \left(-\left(\lambda(x-\gamma-\mu)^{2}\right) /\left(2 \mu^{2}(x-\gamma)\right)\right), \gamma<x<+\infty$ \\
\hline Burr & $\alpha k(x / \beta)^{\alpha-1} /\left(\beta\left(1+(x / \beta)^{\alpha}\right)^{k+1}\right), 0 \leq x<+\infty$ \\
\hline $\log N$ & $\exp \left(-0.5((\ln x-\mu) / \sigma)^{2}\right) /\left(x \sigma(2 \pi)^{0.5}\right), 0<x<+\infty$ \\
\hline Beta & $(x-a)^{\alpha_{1}-1}(b-x)^{\alpha_{2}-1} /\left(B\left(\alpha_{1}, \alpha_{2}\right)(b-a)^{\alpha_{1}}+\alpha_{2}^{-1}\right), a \leq x \leq b, B$ is the Beta Function; $a, b$ is the boundary \\
\hline
\end{tabular}

Table 2 Geographical information and maximum and mode tidal current speeds at the studied locations

\begin{tabular}{cccccccc}
\hline \hline \multirow{2}{*}{ Site Code } & \multirow{2}{*}{ Site Name } & \multicolumn{2}{c}{ Geographic Coordinates } & \multicolumn{4}{c}{ Current Speed (knots) } \\
& & Latitude $(\mathrm{N})$ & Longitude $(\mathrm{W})$ & Max & Mod & Max & Mod \\
\hline 1 & Golden Gate Bridge & $37.82^{\circ}$ & $122.45^{\circ}$ & 4.35 & 2.14 & 4.31 \\
2 & Bahía Honda Harbor & $24.66^{\circ}$ & $81.29^{\circ}$ & 2.66 & 1.27 & 3.34 & 2.79 \\
3 & Daniel Island Reach & $32.83^{\circ}$ & $79.93^{\circ}$ & 1.69 & 1.10 & 2.48 & 1.74 \\
4 & Goodnews Bay entrance & $59.01^{\circ}$ & $161.78^{\circ}$ & 3.53 & 2.04 & 3.17 & 2.36 \\
5 & Heikish Narrows & $52.87^{\circ}$ & $128.50^{\circ}$ & 4.19 & 2.20 & 4.52 & 2.84 \\
6 & Delaware Bay Entrance & $38.78^{\circ}$ & $75.04^{\circ}$ & 2.21 & 1.40 & 2.08 & 1.43 \\
7 & Balch Passage & $47.19^{\circ}$ & $122.70^{\circ}$ & 2.35 & 1.16 & 4.05 \\
8 & Coast Guard Tower & $35.76^{\circ}$ & $75.53^{\circ}$ & 1.93 & 1.31 & 2.84 \\
9 & Nushagak Bay entrance & $58.57^{\circ}$ & $158.42^{\circ}$ & 3.92 & 2.8 & 3.53 \\
10 & Grand Manan Channel & $44.75^{\circ}$ & $66.93^{\circ}$ & 3.99 & 2.47 & 3.78 \\
\hline \hline
\end{tabular}

Table 3 Goodness-of-fit results of different probability distributions for the tidal current speeds at the ten sites (F: flood; E: ebb)

\begin{tabular}{|c|c|c|c|c|c|c|c|c|c|c|c|c|}
\hline Site & & $\mathrm{C}$-Value & WAK & KSD & GEV & GG-3 & Wei-3 & Ray & IG-3 & Burr & $\log N$ & Beta \\
\hline \multirow[b]{2}{*}{1} & $\mathrm{~F}$ & 0.00976 & $0.00391(1)$ & $0.01318(2)$ & $0.02948(3)$ & $0.10993(9)$ & $0.03666(4)$ & $0.06349(7)$ & $0.04131(5)$ & $0.0685(8)$ & $0.14193(10)$ & $0.04314(6)$ \\
\hline & E & 0.00941 & $0.00529(1)$ & $0.01580(2)$ & $0.03031(4)$ & $0.06995(9)$ & $0.033198(5)$ & $0.05242(8)$ & $0.0523(7)$ & $0.0394(6)$ & $0.10792(10)$ & $0.02368(3)$ \\
\hline \multirow{2}{*}{2} & $\mathrm{~F}$ & 0.01011 & $0.00499(1)$ & $0.01482(2)$ & $0.02706(4)$ & $0.07074(8)$ & $0.02442(3)$ & $0.03767(7)$ & $0.04958(8)$ & $0.03272(6)$ & $0.105(10)$ & $0.02864(5)$ \\
\hline & E & 0.00915 & $0.00731(1)$ & $0.01427(2)$ & $0.03352(3)$ & $0.12117(9)$ & $0.04399(4)$ & $0.07199(7)$ & $0.04438(5)$ & $0.07805(8)$ & $0.14877(10)$ & $0.05493(6)$ \\
\hline \multirow{2}{*}{3} & $\mathrm{~F}$ & 0.0098 & $0.00791(1)$ & $0.01058(2)$ & $0.02928(3)$ & $0.12174(9)$ & $0.03819(4)$ & $0.08697(8)$ & $0.04125(5)$ & $0.07247(7)$ & $0.15162(10)$ & $0.05169(6)$ \\
\hline & E & 0.00943 & $0.00825(1)$ & $0.01510(2)$ & $0.03586(3)$ & $0.12161(9)$ & $0.04409(4)$ & $0.07376(7)$ & $0.04636(5)$ & $0.07872(8)$ & $0.14686(10)$ & $0.06071(6)$ \\
\hline \multirow{2}{*}{4} & $\mathrm{~F}$ & 0.00955 & $0.00583(1)$ & $0.01275(2)$ & $0.02798(3)$ & $0.10714(9)$ & $0.03616(4)$ & $0.05883(7)$ & $0.03693(5)$ & $0.06223(8)$ & $0.14012(10)$ & $0.03973(6)$ \\
\hline & E & 0.00958 & $0.00526(1)$ & $0.00694(2)$ & $0.02689(3)$ & $0.1538(9)$ & $0.0365(4)$ & $0.12238(8)$ & $0.07255(6)$ & $0.09794(7)$ & $0.17979(10)$ & $0.04312(5)$ \\
\hline \multirow{2}{*}{5} & $\mathrm{~F}$ & 0.00986 & $0.00509(1)$ & $0.01688(2)$ & $0.03243(4)$ & $0.08424(9)$ & $0.03619(5)$ & $0.05248(8)$ & $0.05168(7)$ & $0.04593(6)$ & $0.11456(10)$ & $0.02512(3)$ \\
\hline & E & 0.00677 & $0.00334(1)$ & $0.01508(2)$ & $0.03162(3)$ & $0.09067(8)$ & $0.03418(5)$ & $0.05133(8)$ & $0.04854(6)$ & $0.05075(7)$ & $0.12179(10)$ & $0.03242(4)$ \\
\hline \multirow{2}{*}{6} & F & 0.0097 & $0.00605(1)$ & $0.01442(2)$ & $0.03226(3)$ & $0.10832(9)$ & $0.0403(4)$ & $0.06396(7)$ & $0.04256(5)$ & $0.06586(8)$ & $0.13692(10)$ & $0.05038(6)$ \\
\hline & E & 0.00955 & $0.0073(1)$ & $0.01291(2)$ & $0.0304(3)$ & $0.11314(9)$ & $0.03904(4)$ & & $0.04179(5)$ & $0.06713(7)$ & $0.14403(10)$ & $0.05278(6)$ \\
\hline \multirow{2}{*}{7} & F & 0.01021 & 0.005 & $0.01694(2)$ & $0.03075(5)$ & $0.067(9)$ & $0.03023(4)$ & 0.046 & $0.05095(8)$ & $0.03529(6)$ & $0.10238(10)$ & (3) \\
\hline & $\mathrm{E}$ & 0.00909 & $0.00528(1)$ & $0.01358(2)$ & $0.02825(3)$ & $0.08742(9)$ & $0.03129(4)$ & $0.04207(6)$ & $0.04617(7)$ & $0.04782(8)$ & $0.11928(10)$ & $0.3148(5)$ \\
\hline \multirow{2}{*}{8} & $\mathrm{~F}$ & 0.01062 & $0.00751(1)$ & $0.01208(2)$ & $0.0288(3)$ & $0.12472(9)$ & $0.03815(4)$ & $0.08961(8)$ & $0.04568(5)$ & $0.07493(7)$ & $0.15563(10)$ & $0.04852(6)$ \\
\hline & E & 0.00882 & $0.00862(1)$ & $0.01514(2)$ & $0.03749(3)$ & $0.12054(9)$ & $0.04453(4)$ & $0.07151(7)$ & $0.04804(5)$ & $0.07739(8)$ & $0.14778(10)$ & $0.06001(6)$ \\
\hline \multirow{2}{*}{9} & $\mathrm{~F}$ & 0.00933 & $0.00576(1)$ & $0.01206(2)$ & $0.02789(3)$ & $0.1074(9)$ & $0.03557(4)$ & $0.05944(7)$ & $0.03605(5)$ & $0.06405(8)$ & $0.13974(10)$ & $0.04019(6)$ \\
\hline & E & 0.00981 & $0.0065(1)$ & $0.00720(2)$ & $0.02748(3)$ & $0.1565(9)$ & $0.03623(4)$ & $0.12278(8)$ & $0.07385(6)$ & $0.09929(7)$ & $0.18178(10)$ & $0.04335(5)$ \\
\hline \multirow{2}{*}{10} & $\mathrm{~F}$ & 0.00949 & $0.00484(1)$ & $0.01626(2)$ & $0.03245(3)$ & $0.09529(9)$ & $0.03603(4)$ & $0.05179(7)$ & $0.04394(6)$ & $0.05712(8)$ & $0.12853(10)$ & $0.03624(5)$ \\
\hline & E & 0.00967 & $0.00354(1)$ & $0.01506(2)$ & $0.03117(3)$ & $0.09113(9)$ & $0.03641(5)$ & $0.04898(7)$ & $0.04605(6)$ & $0.05509(8)$ & $0.12247(10)$ & $0.03276(4)$ \\
\hline
\end{tabular}


Table 4 RMSE indices between histograms and probability density distributions of the tidal current speeds at the ten sites in the posteriori test (F: flood; E: ebb)

\begin{tabular}{|c|c|c|c|c|c|c|c|c|c|c|c|}
\hline Site & & WAK & KSD & GEV & GG-3 & Wei-3 & Ray & IG-3 & Burr & $\log N$ & Beta \\
\hline \multirow[b]{2}{*}{1} & $\bar{F}$ & $0.00167(1)$ & $0.00539(4)$ & $0.00362(2)$ & $0.00863(7)$ & $0.00420(3)$ & $0.00590(5)$ & $0.02201(9)$ & $0.00602(6)$ & $0.01115(8)$ & $0.04304(10)$ \\
\hline & $\mathrm{E}$ & $0.00183(1)$ & $0.00621(7)$ & $0.00359(3)$ & $0.00611(6)$ & $0.00341(2)$ & $0.00490(5)$ & $0.02218(9)$ & $0.00392(4)$ & $0.00904(8)$ & $0.04190(10)$ \\
\hline \multirow{2}{*}{2} & $\mathrm{~F}$ & $0.00183(1)$ & $0.00587(6)$ & $0.00315(3)$ & $0.00598(7)$ & $0.00310(2)$ & $0.00388(5)$ & $0.02206(9)$ & $0.00356(4)$ & $0.00886(8)$ & $0.03023(10)$ \\
\hline & $\mathrm{E}$ & $0.00178(1)$ & $0.00569(4)$ & $0.00419(2)$ & $0.00922(7)$ & $0.00486(3)$ & $0.00667(5)$ & $0.02173(9)$ & $0.00680(6)$ & $0.01151(8)$ & $0.03556(10)$ \\
\hline \multirow{2}{*}{3} & $\mathrm{~F}$ & $0.00177(1)$ & $0.00495(4)$ & $0.00389(2)$ & $0.00947(7)$ & $0.00439(3)$ & $0.00699(6)$ & $0.02233(10)$ & $0.00664(5)$ & $0.01163(8)$ & $0.02211(9)$ \\
\hline & E & $0.00184(1)$ & $0.00579(4)$ & $0.00451(2)$ & $0.00922(7)$ & $0.00517(3)$ & $0.00675(5)$ & $0.02198(9)$ & $0.00687(6)$ & $0.01143(8)$ & $0.02902(10)$ \\
\hline \multirow{2}{*}{4} & $\mathrm{~F}$ & $0.00171(1)$ & $0.00525(4)$ & $0.00339(2)$ & $0.00843(7)$ & $0.00393(3)$ & $0.00566(5)$ & $0.02176(9)$ & $0.00576(6)$ & $0.01092(8)$ & $0.03776(10)$ \\
\hline & E & $0.00152(1)$ & $0.00459(4)$ & $0.00374(3)$ & $0.01141(7)$ & $0.00367(2)$ & $0.00938(6)$ & $0.02279(9)$ & $0.00801(5)$ & $0.01359(8)$ & $0.04553(10)$ \\
\hline \multirow{2}{*}{5} & $\mathrm{~F}$ & $0.00166(1)$ & $0.00623(6)$ & $0.00389(2)$ & $0.00666(7)$ & $0.00397(3)$ & $0.00528(5)$ & $0.02133(9)$ & $0.00460(4)$ & $0.00930(8)$ & $0.03934(10)$ \\
\hline & $\mathrm{E}$ & $0.00186(1)$ & $0.00570(4)$ & $0.00396(2)$ & $0.00810(7)$ & $0.00463(3)$ & $0.00600(6)$ & $0.02147(9)$ & $0.00578(5)$ & $0.01072(8)$ & $0.04285(10)$ \\
\hline \multirow{2}{*}{6} & $\mathrm{~F}$ & $0.00161(1)$ & $0.00560(4)$ & $0.00397(2)$ & $0.00845(7)$ & $0.00454(3)$ & $0.00587(5)$ & $0.02191(9)$ & $0.00597(6)$ & $0.01078(8)$ & $0.02611(10)$ \\
\hline & E & $0.00176(1)$ & $0.00530(4)$ & $0.00382(2)$ & $0.00875(7)$ & $0.00435(3)$ & $0.00606(5)$ & $0.02211(9)$ & $0.00611(6)$ & $0.01109(8)$ & $0.02577(10)$ \\
\hline \multirow{2}{*}{7} & $\mathrm{~F}$ & $0.00190(1)$ & $0.00648(7)$ & $0.00363(3)$ & $0.00599(6)$ & $0.00354(2)$ & $0.00450(5)$ & $0.02109(9)$ & $0.00383(4)$ & $0.00879(8)$ & $0.02546(10)$ \\
\hline & E & $0.00182(1)$ & $0.00556(6)$ & $0.00336(2)$ & $0.00699(7)$ & $0.00359(3)$ & $0.00467(5)$ & $0.02227(9)$ & $0.00453(4)$ & $0.00975(8)$ & $0.04115(10)$ \\
\hline \multirow{2}{*}{8} & $\mathrm{~F}$ & $0.00171(1)$ & $0.00486(4)$ & $0.00378(2)$ & $0.00969(7)$ & $0.00423(3)$ & $0.00722(6)$ & $0.02238(9)$ & $0.00674(5)$ & $0.01188(8)$ & $0.02762(10)$ \\
\hline & E & $0.00185(1)$ & $0.00580(4)$ & $0.00455(2)$ & $0.00919(7)$ & $0.00521(3)$ & $0.00677(5)$ & $0.02200(9)$ & $0.00686(6)$ & $0.01144(8)$ & $0.03180(10)$ \\
\hline \multirow{2}{*}{9} & $\mathrm{~F}$ & $0.00182(1)$ & $0.00529(4)$ & $0.00346(2)$ & $0.00844(7)$ & $0.00400(3)$ & $0.00570(5)$ & $0.02176(9)$ & $0.00583(6)$ & $0.01093(8)$ & $0.04013(10)$ \\
\hline & $\mathrm{E}$ & $0.00154(1)$ & $0.00458(4)$ & $0.00384(3)$ & $0.01158(7)$ & $0.00373(2)$ & $0.00948(6)$ & $0.02282(9)$ & $0.00816(5)$ & $0.01377(8)$ & $0.04833(10)$ \\
\hline \multirow{2}{*}{10} & $\mathrm{~F}$ & $0.00163(1)$ & $0.00599(6)$ & $0.00384(2)$ & $0.00762(7)$ & $0.00424(3)$ & $0.00554(5)$ & $0.02154(9)$ & $0.00535(4)$ & $0.01020(8)$ & $0.03891(10)$ \\
\hline & E & $0.00200(1)$ & $0.00599(6)$ & $0.00387(2)$ & $0.00737(7)$ & $0.00420(3)$ & $0.00533(5)$ & $0.02167(9)$ & $0.00515(4)$ & $0.00992(8)$ & $0.03780(10)$ \\
\hline
\end{tabular}

Table 5 RMSE indices between histograms and theoretical probability distributions at the ten sites

\begin{tabular}{ccccccc}
\multicolumn{7}{c}{ in the priori test (F: flood, E: ebb) } \\
\hline \hline & WAK & E & F & E & F & E \\
\hline 1 & 0.0014 & 0.0014 & 0.0810 & 0.0262 & 0.0438 & 0.0413 \\
2 & 0.0016 & 0.0015 & 0.0749 & 0.0998 & 0.0432 & 0.0443 \\
3 & 0.0015 & 0.0015 & 0.1001 & 0.1001 & 0.0476 & 0.0449 \\
4 & 0.0017 & 0.0016 & 0.0967 & 0.1001 & 0.0445 & 0.0480 \\
5 & 0.0012 & 0.0012 & 0.0423 & 0.0517 & 0.0423 & 0.0424 \\
6 & 0.0015 & 0.0015 & 0.0450 & 0.1001 & 0.0450 & 0.0458 \\
7 & 0.0016 & 0.0014 & 0.0430 & 0.0464 & 0.0430 & 0.0433 \\
8 & 0.0016 & 0.0015 & 0.0473 & 0.1001 & 0.0473 & 0.0441 \\
9 & 0.0016 & 0.0016 & 0.0441 & 0.1002 & 0.0441 & 0.0481 \\
10 & 0.0011 & 0.0013 & 0.0671 & 0.0681 & 0.0426 & 0.0434 \\
\hline \hline
\end{tabular}

\section{Figure Captions}

Figure 1 PDF plots and histogram of flood current speed at site 4

Figure 2 PDF plots and histogram of the flood current speed at site 6

Figure 3 PDF plots and histogram of the ebb current speed at site 8

Figure 4 PDF plots and histogram of the ebb current speed at site 9

Figure 5 Differences between the Wakeby distribution samples and actual flood current speed data at site 10

Figure 6 Differences between the Wakeby distribution samples and actual ebb current speed data at site 10 


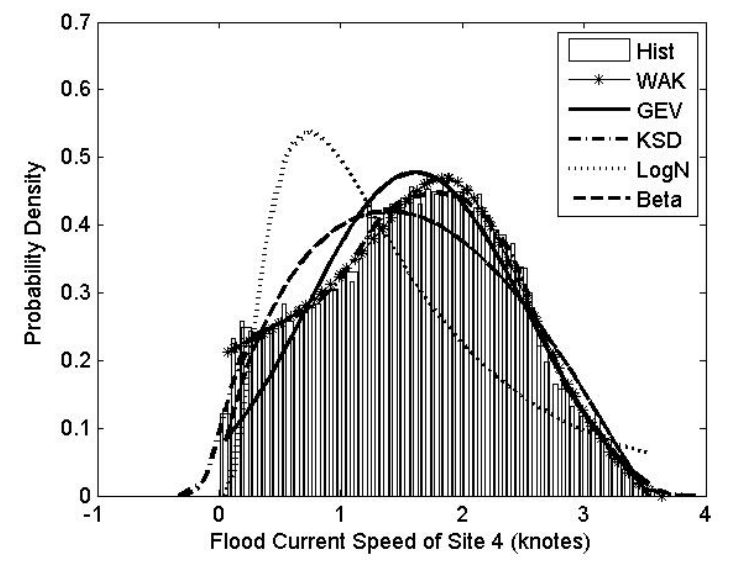

Figure 1 PDF plots and histogram of flood current speed at site 4

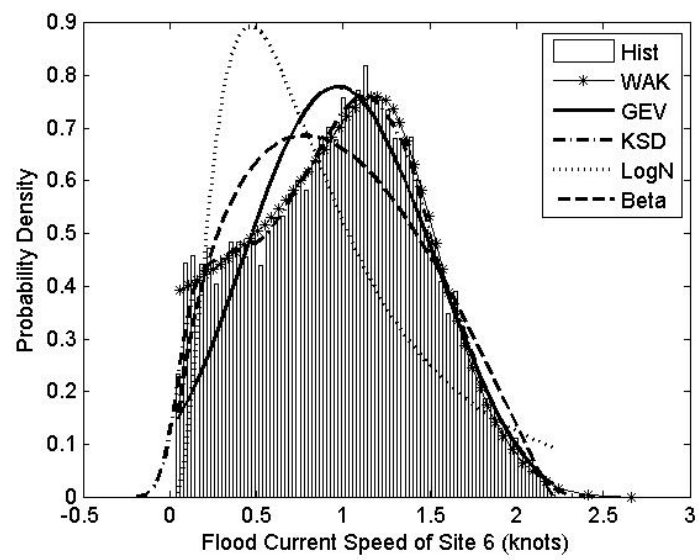

Figure 2 PDF plots and histogram of the flood current speed at site 6

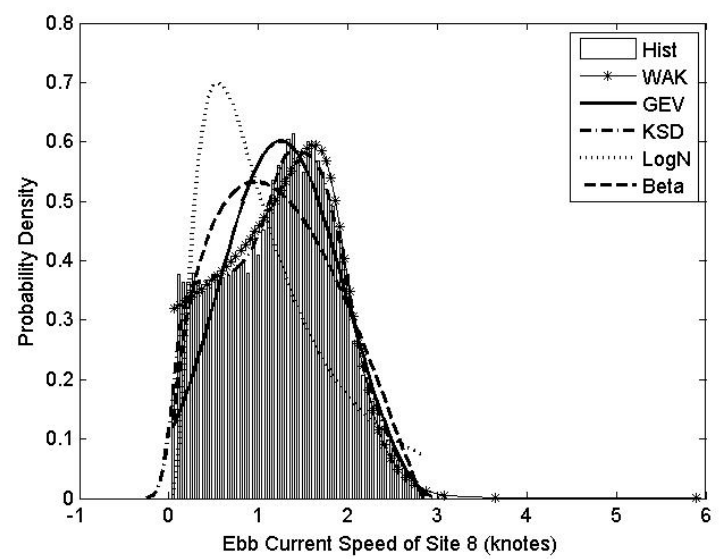

Figure 3 PDF plots and histogram of the ebb current speed at site 8

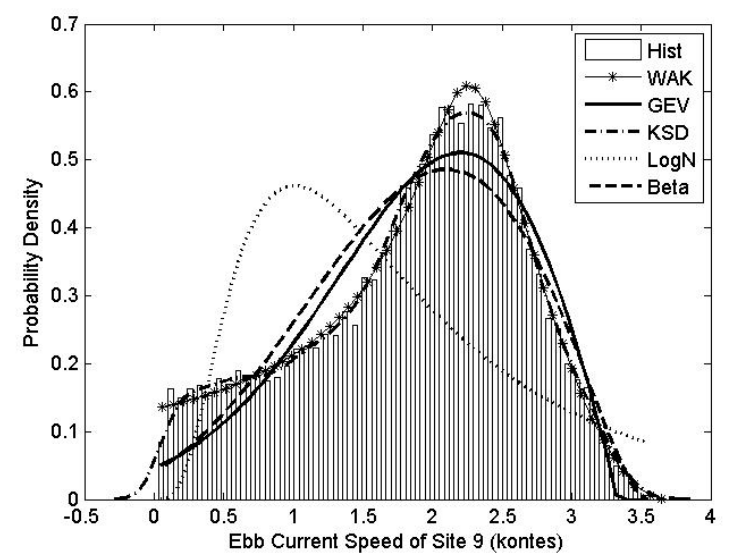

Figure 4 PDF plots and histogram of the ebb current speed at site 9

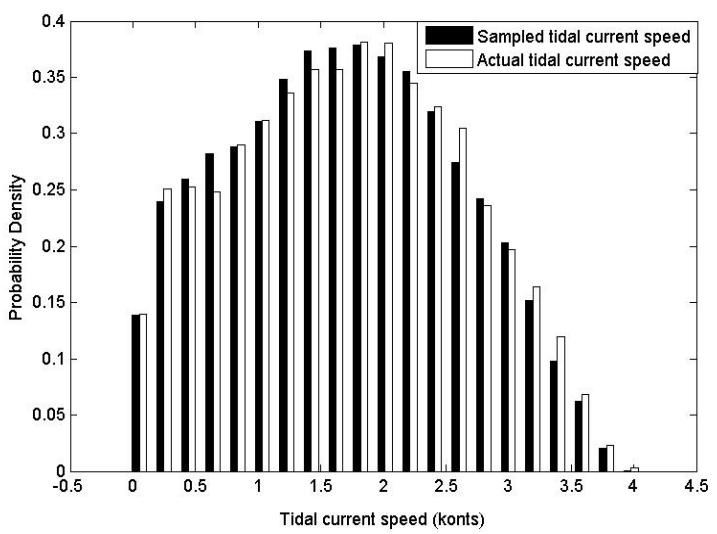

Figure 5 Differences between the Wakeby distribution samples and actual flood current $\begin{array}{lll}\text { speed data at } & \text { site }\end{array}$

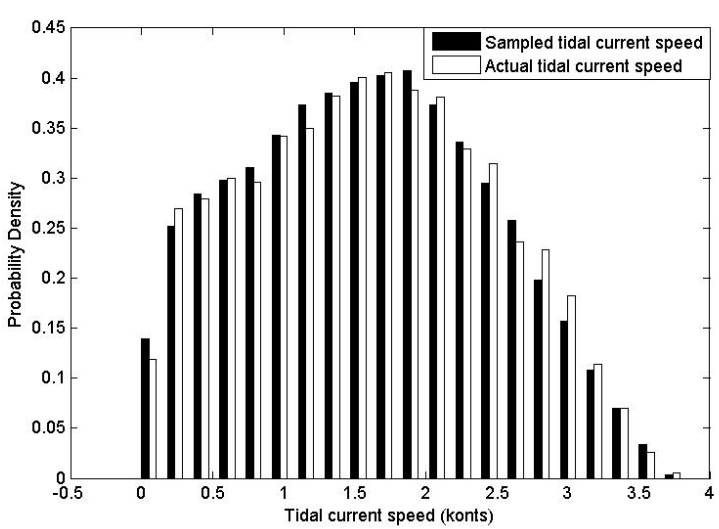

Figure 6 Differences between the Wakeby distribution samples and actual ebb current speed data at site 10 
Dr. Caisheng Wang received the B.S. and M.S. degrees from Chongqing University, China, in 1994 and 1997, respectively, and the Ph.D. degree from Montana State

\section{Biography}

Mr. Mingjun Liu is currently working toward the Ph.D. degree in the School of 
University, Bozeman, MT, in 2006, all in electrical engineering. From August 1997 to May 2002, he was an Electrical Engineer and then a Vice-Department Chair with Zhejiang Electric Power Test and Research Institute, Hangzhou, China. Since August 2006, he has been with Wayne State University, Detroit, MI, where he is currently an Associate Professor in the Department of Electrical and Computer Engineering. His current research interests include modeling and control of power systems and electrical machines, energy storage devices, hybrid energy power generation systems, fault diagnosis, and online monitoring of electric apparatus.

Dr. Juan Yu received the Ph.D. degree in electrical engineering from Chongqing University, Chongqing, China, in 2007. Currently, she is a professor in the School of Electrical Engineering at Chongqing University. Her research interests include reactive optimal problem, static equivalent, voltage stability and risk assessment in power systems. 